\title{
A global view of behavioural design approach for 3D printing in the early design stage
}

\author{
JIANG Yixue ${ }^{1, \text { a }}$, SUN Huichao, ${ }^{*, \mathrm{~b}}$, JI Shijiun ${ }^{2, \mathrm{c}}$, ZHAO Liying $^{1, \mathrm{~d}}$, ZHAO \\ Yaohong ${ }^{3, e}$ \\ ${ }^{1}$ Computer Foundation Teaching Center, Changchun Institute of Technology, China \\ ${ }^{2}$ School of Mechanical Science and Engineering , Jilin University, China \\ ${ }^{3}$ Faculty of computer science and technology, Changchun University, China \\ a124621823@qq.com, bsunhc@jlu.edu.cn, cjishijun97@126.com, d397461267@qq.com, \\ e234581000@qq.com
}

\begin{abstract}
Keywords: product design behavioural design, utilization condition, utilization requirement, 3D printing
\end{abstract}

\begin{abstract}
The mechanical engineering problems today become more and more complex particularly in the area of new product development. Mechanical product design is usually performed simply taking into consideration system functions and structures, while users' behaviours in terms of using the system are generally not fully considered during the early design phase. This paper covers the multi-trade engineering design, and deals with the development of a behavioural design approach to help designers to optimize the product performance in the early design phase through taking into account utilization conditions and requirements.
\end{abstract}

\section{Introduction}

Mechanical product design processes are often technology-centered and fail to integrate user's behaviour in term of using the product adequately. This problem is encountered along the whole life cycle of a project, and is especially noticeable during the early design phase. These behaviours take place all over the product lifecycle. In order to improve product performance, our research carefully thinks out a piece of research linking user centered and functional engineering design approached into an integrated package, and aims to better integrate product and user behaviour during the early design phase. Designers have been obliged to set aside their dreams of a $100 \%$ machine due to the vital requirement of the user to perform some definite tasks with machines. While machine productivity and utilization conditions are the main reasons for automating production systems, human intervention on such systems remains a critical need and the tasks performed by the user remain poorly defined at the early design stage.

In traditional engineering design, designers normally take into consideration product functions and structures, while users' behaviours in terms of using the system are generally not fully considered during the early design phase. A product's behaviour is studied only from a technical point of view in order to verify its reliability and potential problems in the detailed design phase. However, this behaviour is neither characterised nor studied from a utilization point of view. Nowadays, al-though designers do increasingly have some understanding of user behaviour, they rarely pay much attention to the behaviour which derives from the structure (how the structure will move to fulfil the function), and behaviour which is fulfilled by the user (how the user will react to the machine).

It is known that the user's perception of a system is quite different from the designer's [1]. Additionally, involving a range of users in design by adopting an inclusive approach has been identified as an important way through which companies can manufacture more successful systems [2-3]. To separate system technology from user-related features, it is necessary to split the notion of system into two separate components: technical solutions and user-related features [4]. The strategy of knowledge management is not widely adopted for innovation in industries due to a lack of an effective approach of integration between user knowledge and technical knowledge [5]. Most current 
technical approaches stop at the functional level, without analyzing how the overall system (system-user) could behave in perform these functions.

The objective of this paper is to propose an approach to help the designer optimize product performance from the early design phase, taking into account use conditions and requirements. This approach is based on a Task Model and on the fact that the behavioural system (system and end-user) must be studied and defined from the early design phase. We focus on a production system design, and so, to complete the mechanical system design method, we propose a global view of behavioural design approach in the early design stage.

\section{BEHAVIOURAL DESIGN APPROACH (BDA) for 3D printing}

According to the real industry examples and references reviewed above, we conclude that there are two aspects to the concept of behaviour. The first involves behaviour which is carried out by the system according to the technical viewpoints. The second involves behaviour which is carried out by the users of the system or the correlative working team.

According to the proposed behavioural design approach, on one hand, designers find out the technical solutions to fulfill some of the technical functions defined in the functional analysis. On the other hand, when designers do not find feasible technical solutions for the other functions, or due to cost reasons, they propose to fulfill these functions by user task (correlative working team).

We herein propose the Behavioural Design Approach (BDA) to integrate user behaviours and structure behaviours from the early design phase. Behavioural design is a mechanical product design method based on multidisciplinary knowledge that takes into account, from its preliminary phases, the analysis and the specification of utilization tasks necessary for accomplishing the functions [8]. We propose below the global view of the behavioural design approach. It represents a set of steps linking together the three concepts (function, structure, and behaviour), as shown in Figure 1.

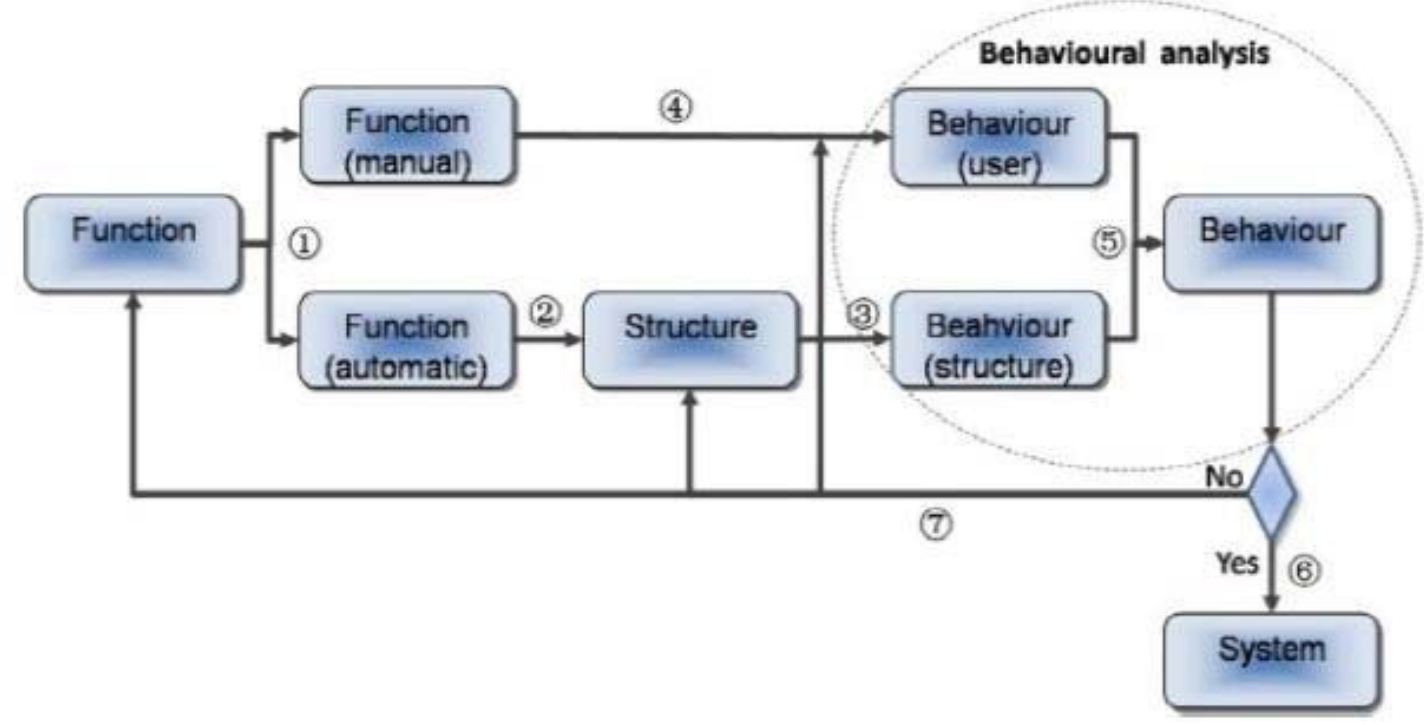

Figure 1 Global view of the behavioural design approach for 3D printing

Seven steps are listed here to describe the modelling procedure:

Step 1: According to the Functional Analysis and requirements specification, we can divide the function into two parts. The first is the automatic function realized by technical solutions; the second is the manual function fulfilled by the user, because of the cost or the difficulties related to automation.

Step 2: According to some methods, such as FAST [9], Axiomatic Design[10], we could find the necessary structure to carry out the function.

Step 3: According to structure decomposition, we can obtain the behaviour of structure tasks (operation, motion, etc.) that the structure has to perform to achieve the function. 
Step 4: Manual functions will be carried out by the user. Thus, in this step we propose identifying and studying the tasks performed by the user to fulfil manual functions.

Step 5: To improve the performance of the system, we propose that the

interaction between the structure's behaviour as well as the user's be analysed.

Step 6: If the structure's behaviour meets the performance criteria (functionality, productivity, safety, cost, quality, etc.), designers can continue to develop the system.

Step 7: Where the interaction between the user's behaviour and that of the

structure does not ensure the needed performance, we have to change user's tasks, or go back to the structure level to modify the structure or go back to the function level to modify or change the function decomposition. We could also change the task performed by the user, which means changing the user's behaviour.

The BDA enters from these design steps to help designers to classify the manual function and automatic function. And then our software help designer to obtain the structure behaviour which are derived from embodiment design (structure analysis). The BDA also can analyze the user behaviour which is derived from the manual function. And then in the detail design, it includes specifying the materials, the sizes and so on. All these factors are determined by both the technical solutions and the socio-technical solutions which are influenced by the integration of structure behaviour and user behaviour. The BDA will finally determine the integration of these two types of behaviour which can aid the designer to finish the system build. The BDA can help designers to find the potential dangerous factors before the manufacturing phase which approves the performance and reduce the cost of the redesign.

\section{CONCLUSION AND PERCEPTIVE}

This paper mainly focuses on the field of the mechanical product design and a better integration of utilization requirements in order to improve the performance of product. The major improvements are based on the behavioural analysis compared with the traditional approaches of design, which is realized very late to satisfy the requirements of standards and proposes some difficult procedures to do by user. This lately integration causes a degradation of system performance, which generates a variation between what is imagined by the designers and what is realized by the user.

This paper presents the behavioural design to well integrate with the using tasks of production system and more particularly user's behaviour in the early design phase. The task concept that we proposed to define behavior is presented. Our actual work deal with two issues: (1)to clarify the structure's behaviour in use and the response of its user, and to identify the interactions between the structure"s behaviour and user's behaviour; (2)to accomplish the global view of behavioural design approach including both technical viewpoints and human viewpoints. In the future work, we will take into count the applicability of our approach and its consequence on designer's work. A software using SysML is currently in development to support and allow a systematic utilization of the "behavioural design" by integrating it in the daily work of the designer.

\section{Acknowledgements}

This work was financially supported by the National Natural Science Foundation of China (51305161).

\section{References}

[1]. Battini, D., Faccio, M., Personaa, A ., Sgarbossaa, F., 2011. "New methodologicalframework to improve productivity and ergonomics in assembly system design." International Journal of Industrial Ergonomics 41 (1), 30-42.

[2]. Gyi, D., R. Cain, et al. 2010. "The value of computer-based product representations in co-designing with older users." Journal of Engineering Design 21(2): 305-313. 
[3]. Huisg, S. and S. Kohn 2009. "Computer aided innovation--State of the art from a new product development perspective." Computers in Industry 60(8): 551-562.

[4]. Sun, H., R. Houssin, et al. 2010. «La conception comportementale : Une nouvelle approche pour améliorer la performance de produit.» 17ème Congrès de Maîtrise des Risques et de Sûreté de Fonctionnement Gentilly, France.

[5]. European Communities. 2009. "Design as a driver of user-centered design." Commision of staff working document.

[6]. Bagge, S. and A. M. Pendrill 2002. "Classical physics experiments in the amusement park." Physics Education 37: 507.

[7]. Houssin, R., A. Bernard, et al. 2006. "Information system based on a working situation model for a new design approach in concurrent engineering." Journal of Engineering Design 17(1): 35-54.

[8]. Sun, H., R. Houssin, et al. 2011. "Improving Product Performance With Integration Of Using Tasks During The Design Phase: A Behavioural Design Approach. " International Conference on Industrial Engineering and Systems Management. METZ FRANCE.

[9]. Wixson, J. R. 1999. "Function analysis and decomposistion using function analysis systems techniqu." Citeseer.

[10]. Suh, N. P. 2000. “Axiomatic design.” Oxford university press. 